$\stackrel{N / N}{=}$

Global burnals Inc.

औf
GLOBAL JOURNAL OF MEDICAL RESEARCH: A

NEUROLOGY \& NERVOUS SYSTEM

Volume 21 Issue 3 Version 1.0 Year 2021

Type: Double Blind Peer Reviewed International Research Journal

Publisher: Global Journals

Online ISSN: 2249-4618 \& Print ISSN: 0975-5888

\title{
The Effect of Cognitive Behaviour Therapy on Sleep and Circadian Rhythm in Young College Students
}

\author{
By Shweta Kanchan, Sunita Tiwari \& Shweta Singh
}

Abstract- The present study is to study the effect of cognitive behaviour therapy on various sleep parameters and circadian phase rhythmic in young college going adults. Fifty young college going adults were chosen from the MBBS and BDS students of King George's Medical University Lucknow, their polysomnography was conducted along with it salivary melatonin estimation was conducted to find the time of Dim light melatonin onset (DLMO), the subjects were administered cognitive behaviour therapy (CBT), after completing the sessions of cognitive behaviour therapy another Polysomnographic study and DLMO estimation was conducted, various sleep parameters were compared before and after the CBT. The study showed an improvement in the steep quality, a decrease in daytime sleepiness along with this total sleep time significantly increased, sleep efficiency also improved and there was a decrease in the REM sleep latency. The Dim light melatonin onset advanced for the subjects and the chronotype showed an inclination towards an earlier timings.

Keywords: cognitive behavior therapy (CBT), pittsburgh sleepiness scale (PSQI), epworth sleepiness scale (ESS), dim light melatonin onset (DLMO).

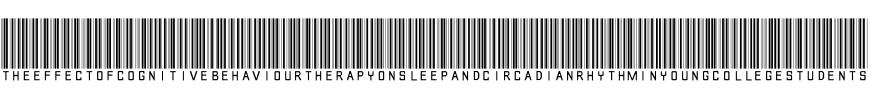

Strictly as per the compliance and regulations of:

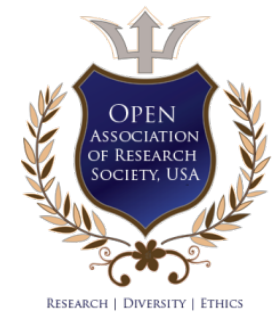

(c) 2021. Shweta Kanchan, Sunita Tiwari \& Shweta Singh. This is a research/review paper, distributed under the terms of the Creative Commons Attribution-Noncommercial 3.0 Unported License http://creativecommons.org/licenses/by-nc/3.0/), permitting all non-commercial use, distribution, and reproduction in any medium, provided the original work is properly cited. 


\title{
The Effect of Cognitive Behaviour Therapy on Sleep and Circadian Rhythm in Young College Students
}

\author{
Shweta Kanchan ${ }^{\alpha}$, Sunita Tiwari $^{\circ}$ \& Shweta Singh ${ }^{\rho}$
}

\begin{abstract}
The present study is to study the effect of cognitive behaviour therapy on various sleep parameters and circadian phase rhythmic in young college going adults. Fifty young college going adults were chosen from the MBBS and BDS students of King George's Medical University Lucknow, their polysomnography was conducted along with it salivary melatonin estimation was conducted to find the time of Dim light melatonin onset (DLMO), the subjects were administered cognitive behaviour therapy (CBT), after completing the sessions of cognitive behaviour therapy another Polysomnographic study and DLMO estimation was conducted, various sleep parameters were compared before and after the CBT. The study showed an improvement in the steep quality, a decrease in daytime sleepiness along with this total sleep time significantly increased, sleep efficiency also improved and there was a decrease in the REM sleep latency. The Dim light melatonin onset advanced for the subjects and the chronotype showed an inclination towards an earlier timings.
\end{abstract}

Keywords: cognitive behavior therapy (CBT), pittsburgh sleepiness scale (PSQI), epworth sleepiness scale (ESS), dim light melatonin onset (DLMO).

\section{INTRODUCTION}

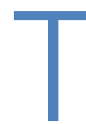
he present study was conducted to find out the efficacy of cognitive behaviour therapy on different sleep parameters and circadian phase timings in college students in the age group of 18 to 25 years. Young college going adults are more prone to delay their sleep and report poor sleep quality because of the demand of career and social life in a highly competitive environment. Several previous studies have shown that adequate quantity and quality of sleep is required for optimal physiological functions (1). Young adults suffer from several short term and long term consequences due to sleep lack in the form of excessive daytime sleepiness, irritability, cognitive impairment, depression, low immunity, metabolic disorders (2). The present study tries to understand the effects of cognitive behaviour therapy in improving their sleep parameters and circadian phase advancement.

Author a: e-mail: drshwetakanchan@gmail.com

\section{il. Methodology}

The present study was conducted on students of MBBS and BDS in King George's Medical University, Lucknow. A screening questionnaire based on Pittsburgh Sleep Quality Index (PSQI) (3) was served initially, this questionnaire gives information about the sleep quality and from all those who scored more than 7 on the PSQI and consented to participate, subjects were randomly selected using random number table and finally 50 subjects were recruited for the study. The participants selected were given epworth sleepiness scale to find out the degree of daytime sleepiness. A score more than 10 in the epworth sleepiness scale (ESS) (4) indicates significant degree of daytime sleepiness. The subjects were provided with sleep diaries which had to be filled for 14 days after which an overnight polysomnographic study of subject was conducted using SOMNOscreen plus EEG32 video equipped polysomnography with a resolution of 16 bit, sampling rate up to $512 \mathrm{~Hz}$, and band pass filter of 0.1 to $128 \mathrm{~Hz}$. Standard electrode placement for EEG( f4$m 1, c 4-m 1,02-m 1$ along with alternate at $\mathrm{f1}-\mathrm{m} 2, \mathrm{c} 1-$ $m 2,01-m 2)$, chin EMG and other channels according to the recommendations of the AASM were used. Parameters which were considered for the study were sleep latency (SL), sleep efficiency, REM latency, Total Sleep Time(TST). Changes in sleep architecture using the above parameters before and after intervention was compared. Along with the polysomnography circadian rhythm was marked by their salivary melatonin level by collecting salivary samples every 30 min beginning at 9:00 pm till the subject goes to sleep, both sleep study and salivary collection were conducted in the sleep lab on the same night. Subjects were expected to collect saliva in labeled eppendorf tubes, collection was conducted in the sitting position in dim light of less than 30 lux (5), the subjects were refrained to eat in the lab, about 4 to $5 \mathrm{ml}$ of saliva was collected and promptly refrigerated, early in the morning salivary samples were centrifuged at 2000 RPM and the and the supernatant was stored at minus 80 degree and examined when all samples were collected. Human melatonin Elisa kit from Bioassay technology laboratory was used for salivary melatonin estimation, Elisa test was conducted according to the user instruction, a standard curve 
plotting the optical density of each sample and was corellated with salivary melatonin concentration. The Dim light melatonin onset (DLMO) is the time at which the salivary melatonin reached $4 \mathrm{pg} / \mathrm{ml}$, it is considered a useful parameter for estimation of circadian rhythm (6), DLMO time was noted. After completing all sessions of cognitive behavior therapy (CBT), a sleep study was again conducted accompanied with salivary melatonin estimation for circadian phase change using DLMO as a marker was noted. The subjects were given intervention in the form of cognitive behaviour therapy in 4 to 5 session. Cognitive behaviour therapy module were prepared according to the sleep related complains faced by these young students obtained after interviewing them. These models were divided into four weekly sessions of 45 minutes to 1 hour each a fifth session was administered if needed. They were given a sleep diary after the completion of all CBT sessions, while they were expected to comply with the instructions and methods learned during CBT. A morningness eveningness questionnaire was also served both before and after the intervention to find out any changes in the chronotype, PSQI scale and the Epworth Sleepiness Scale, were also served after completion of the study.

\section{Results}

The present study showed that the mean Pittsburgh sleep quality index and the Epworth sleepiness scale slightly decreased post intervention though the changes were not statistically significant (table 1). The total sleep time increased significantly by a mean of 26.43 minutes from the sleep time prior to the intervention. sleep latency however the difference was not statistically significant from $14.70 \mathrm{~min}$ before intervention to $13.94 \mathrm{~min}$ post intervention. Sleep efficiency showed slight improvement from $89.76 \%$ to $92.66 \%$, the changes were more in males than females. REM sleep latency decreased from 123.98 to 109.40 min the mean bed time change was an advance of 123.16 min and the wake up time did not change much, mean wake up time change was $14 \mathrm{~min}$ for the participants and remain somewhere between 7 to $7: 30$ a.m. because the wake up time was mostly decided by their college schedule and they did not have much choice (table 1). The changes in different parameters were nearly same for both the genders however males showed more significant increase in sleep efficiency compared to females (table 2) The dim light melatonin onset (DLMO) advanced by nearly 42 minutes leading to a circadian phase advancement in the post intervention assessment (figure1). The chronotype type as assessed by MEQ score showed no change in the percentage of subjects with a morningness chronotype (18\%), however the subject with intermediate chronotype increased slightly from $74 \%$ to $76 \%$ (table 3 ).

\section{Discussions}

The present study reiterated the findings to several previous study which have found cognitive behaviour therapy to be an effective intervention in advancing phase and also improving sleep quality in young adults. A previous study by Yu jen lee, et all (7) showed that poor academic performance is associated with sleep debt and is represented by extended weekend sleep in case of young college going adults. In the Present study most of the participants had impaired night time sleep in form of less duration of sleep and a poor quality sleep, extended sleep on the weekends along with that they had in poor daytime performance reflected by high scores on the ESS scale, because of insufficient sleep. Most of the participants fulfilled the criteria of behavioral induce insufficient sleep syndrome. Insufficient sleep syndrome has an undiagnosed burden on the gross domestic product and looking at this it has been demanded to declare insufficient sleep a public health epidemic Vijay chatta(8). A study by Stephen Baker(9) the prevalence of mental health problems in college students could link some of the problems to poor sleep the present study also found a very high degree of daytime sleepiness in college students which could be because of the sleep debt that they carry over months, this chronic sleep insufficiency could lead to delayed sleep phase disorder and other health related complains in the long run and should be timely addressed (10). A previous study conducted by Michael Gradiser(11) on the effect of CBT and bright light for the treatment of delayed sleep phase disorder in adolescence showed that CBT is an effective intervention for improving multiple related problems and daytime impairment.

Another study by Zong Rui Ma(12) cognitive behaviour therapy in children and adolescents with insomnia a significant pooled effect size was observed for sleep onset latency and sleep efficiency there was no effect on the wake after sleep onset and other parameters .The present study also observed changes in sleep latency and sleep efficiency after cognitive behavior therapy. Bei Bei et all (13) showed the chronotype in sleep efficiency is associated with sleep outcome the of cognitive behaviour therapy this could explain why the students shift a little from eveningness to intermediate chronotype after the intervention. Melatonin is a very important physiological measure of circadian phase it is not just feasible but also very easy to measure compared to plasma melatonin or urinary melatonin, Henry keijer (14) showed that salivary melatonin is effective measurement of DLMO correlates well with plasma melatonin and urinary melatonin levels. The study used salivary melatonin as a marker of circadian phase. 


\section{Conclusion}

The presence of poor sleep quality and quantity in young adults can lead to poor health in the long run. Cognitive behavior therapy has shown effectiveness in improving sleep and circadian parameters in young adults, it should be encouraged as an effective non pharmacological therapy for better sleep with stress on improving sleep hygine and promoting awareness towards better sleep in the community.

\section{BIBLIOGRAPHY}

1. Orzeł-Gryglewska, J. (2010). Consequences of sleep deprivation. International Journal of Occupational Medicine and Environmental Health, 23(1), 95-114.

2. Faith S. Luyster, PhD, Patrick J. Strollo, Jr., MD, Phyllis C. Zee, MD, PhD, James K. Walsh, PhD, Sleep: A Health Imperative, Sleep, Volume 35, Issue 6, 1 June 2012, Pages 727-734.

3. Daniel J. Buysse, Charles F. Reynolds, Timothy H. Monk, Susan R. Berman, David J. Kupfer, The Pittsburgh sleep quality index: A new instrument for psychiatric practice and research, Psychiatry Research, Volume 28, Issue 2,1989.

4. Murray W. Johns, A New Method for Measuring Daytime Sleepiness: The Epworth Sleepiness Scale, Sleep, Volume 14, Issue 6, November 1991, Pages 540-545.

5. Tomoaki Kozaki, Toshihiko Arata, Ayaka Kubokawa, "Salivary Melatonin Concentrations in a Sitting and a Standing Position", Journal of Hormones, vol. 2013, Article ID 236325, 4 pages, 2013.

6. Attenburrow, M.E.J., Dowling, B.A., Sargent, P.A. et al. Melatonin phase advances circadian rhythm. Psychopharmacology121, 503-505 (1995).

7. Lee YJ, Park J, Kim S, Cho SJ, Kim SJ. Academic performance among adolescents with behaviorally induced insufficient sleep syndrome. J Clin Sleep Med 2015; 11(1):61-68. 6
8. Chattu VK, Sakhamuri SM, Kumar R, Spence DW, BaHammam AS, Pandi-Perumal SR. Insufficient Sleep Syndrome: Is it time to classify it as a major noncommunicable disease? Sleep Sci. 2018 MarApr; 11(2): 56-64.

9. Stephen P. Becker, Matthew A. Jarrett, Aaron M. Luebbe, Annie A. Garner, G. Leonard Burns, Michael J. Kofler, Sleep in a large, multi-university sample of college students: sleep problem prevalence, sex differences, and mental health correlates, Sleep Health, Volume 4, Issue 2, 2018, Pages 174-181

10. Gradisar M, Crowley SJ. Delayed sleep phase disorder in youth. Current Opinion in Psychiatry. 2013 Nov; 26(6):580-585. DOI: 10.1097

11. Michael Gradisar, PhD, et all, A Randomized Controlled Trial of Cognitive-Behavior Therapy Plus Bright Light Therapy for Adolescent Delayed Sleep Phase Disorder, Sleep, Volume 34, Issue 12, 1 December 2011, Pages 1671-1680.

12. Zhong-Rui Ma Li-Jun Shi Ming-Hong Deng Efficacy of cognitive behavioral therapy in children and adolescents with insomnia: a systematic review and meta-analysisBraz. J. Med. Biol. Res. 51 (6) • 2018.

13. Bei Bei, PhD, Jason C. Ong, PhD, Shantha M.W. Rajaratnam, PhD, and Rachel Manber, PhD Chronotype and Improved Sleep Efficiency Independently Predict Depressive Symptom Reduction after Group Cognitive Behavioral Therapy for Insomnia: September 15, 2015 https://doi.org/10.5664/jcsm.5018

14. Henry Keijzre at et all Evaluation of salivary melatonin measurements for Dim Light Melatonin Onset calculations in patients with possible sleepwake rhythm disorders, Clinica Chimica Acta, Volume 412, Issues 17-18, 2011,Pages 1616-1620, ISSN 0009-8981.

Table 1: Descriptive Statistics of All Variables

\begin{tabular}{|c|cccc|}
\hline & N & \multicolumn{3}{c|}{ N=50 } \\
\cline { 2 - 5 } & & Mean & Median & SD \\
\hline AGE Years & 50 & 20.26 & 20.00 & 1.90 \\
TIME SPEND IN INSTT.(months) & 50 & 5.50 & 4.00 & 2.82 \\
BMI & 50 & 22.34 & 22.03 & 2.74 \\
PSQI-Pre & 50 & 11.56 & 11.50 & 1.86 \\
PSQI-Post & 50 & 11.40 & 11.00 & 1.92 \\
ESS-Pre & 50 & 8.90 & 9.00 & 1.57 \\
ESS-Post & 50 & 8.60 & 9.00 & 1.73 \\
TST-Pre & 50 & 297.94 & 300.00 & 22.90 \\
TST-Post & 50 & 324.22 & 325.50 & 23.06 \\
\hline
\end{tabular}




\begin{tabular}{|c|cccc|}
\hline TST-Pre & 50 & 4.97 & 5.00 & 0.38 \\
TST-Post & 50 & 5.40 & 5.43 & 0.38 \\
SLEEP LATENCY-Pre & 50 & 14.70 & 14.50 & 5.25 \\
SLEEP LATENCY -Post & 50 & 13.94 & 14.00 & 5.45 \\
\%Sleep efficiency-Pre & 50 & 89.76 & 89.50 & 3.30 \\
\%Sleep Efficiency-Post & 50 & 92.66 & 94.00 & 3.46 \\
REM Latency-Pre & 50 & 123.98 & 127.00 & 14.73 \\
REM Latency-Post & 50 & 109.40 & 110.00 & 15.04 \\
BedTime Change & 50 & 123.16 & 120.00 & 33.64 \\
WakeUpTime Change & 50 & 14.00 & 15.00 & 14.03 \\
DLMO Time Change & 50 & -42.00 & -40.00 & 26.42 \\
\hline
\end{tabular}

Table 2: Gender Wise Summary of Different Parameters

\begin{tabular}{|c|c|c|c|}
\hline & Sex & $\mathrm{M}$ & $\mathrm{F}$ \\
\hline & $\mathrm{N}$ & 21 & 29 \\
\hline \multirow[t]{4}{*}{ PQSI-Change } & mean & 0 & -0.27586 \\
\hline & sd & 3.11448 & 2.34363 \\
\hline & $p$ & 1 & 0.5312 \\
\hline & N & 29 & 21 \\
\hline \multirow[t]{2}{*}{ ESS-Change } & Mean & 0.45 & 0.10 \\
\hline & $\begin{array}{c}\mathrm{SD} \\
\mathrm{p}\end{array}$ & $\begin{array}{c}2.37 \\
0.1582 \\
\end{array}$ & $\begin{array}{c}2.59 \\
0.8667 \\
\end{array}$ \\
\hline \multirow[t]{3}{*}{ TST-Change } & Mean & 26.17 & 26.43 \\
\hline & SD & 17.89 & 15.55 \\
\hline & $\mathrm{p}$ & $<0.00001$ & $<0.00001$ \\
\hline Sleep Latency- & Mean & -0.34 & -1.33 \\
\hline \multirow[t]{2}{*}{ Change } & SD & 4.34 & 4.18 \\
\hline & $p$ & 0.6705 & 0.16 \\
\hline \multirow[t]{2}{*}{ \%SI.Efficiency } & Mean & 3.24 & 2.43 \\
\hline & $\begin{array}{c}\mathrm{SD} \\
\mathrm{p}\end{array}$ & $\begin{aligned} & 2.86 \\
&< 0.00001 \\
&\end{aligned}$ & $\begin{array}{l}3.53 \\
0.01 \\
\end{array}$ \\
\hline \multirow[t]{3}{*}{ REM-Change } & Mean & -14.21 & -15.10 \\
\hline & SD & 7.04 & 5.53 \\
\hline & $\mathrm{p}$ & $<0.00001$ & $<0.00001$ \\
\hline \multirow[t]{3}{*}{ DLMO CHANGE } & MEAN & -41.38 & -36.92 \\
\hline & SD & 18.46 & 24.66 \\
\hline & $\mathrm{P}$ & $<.0001$ & $<.0001$ \\
\hline
\end{tabular}

Table 3: Morningness-Eveningness Scores (Meq Scores) Pre and Post Cbt Intervention

\begin{tabular}{|l|c|c|c|c|}
\hline EQ SCORE & $\mathrm{N}=50$ & & $\mathrm{~N}=50$ & \\
\hline & Pre(number) & percentage & Post( number) & percentage \\
\hline Morningness & 9 & $18 \%$ & 9 & $18 \%$ \\
\hline Eveningness & 4 & $8 \%$ & 3 & $6 \%$ \\
\hline Intermediate & 37 & $74 \%$ & 38 & $76 \%$ \\
\hline
\end{tabular}




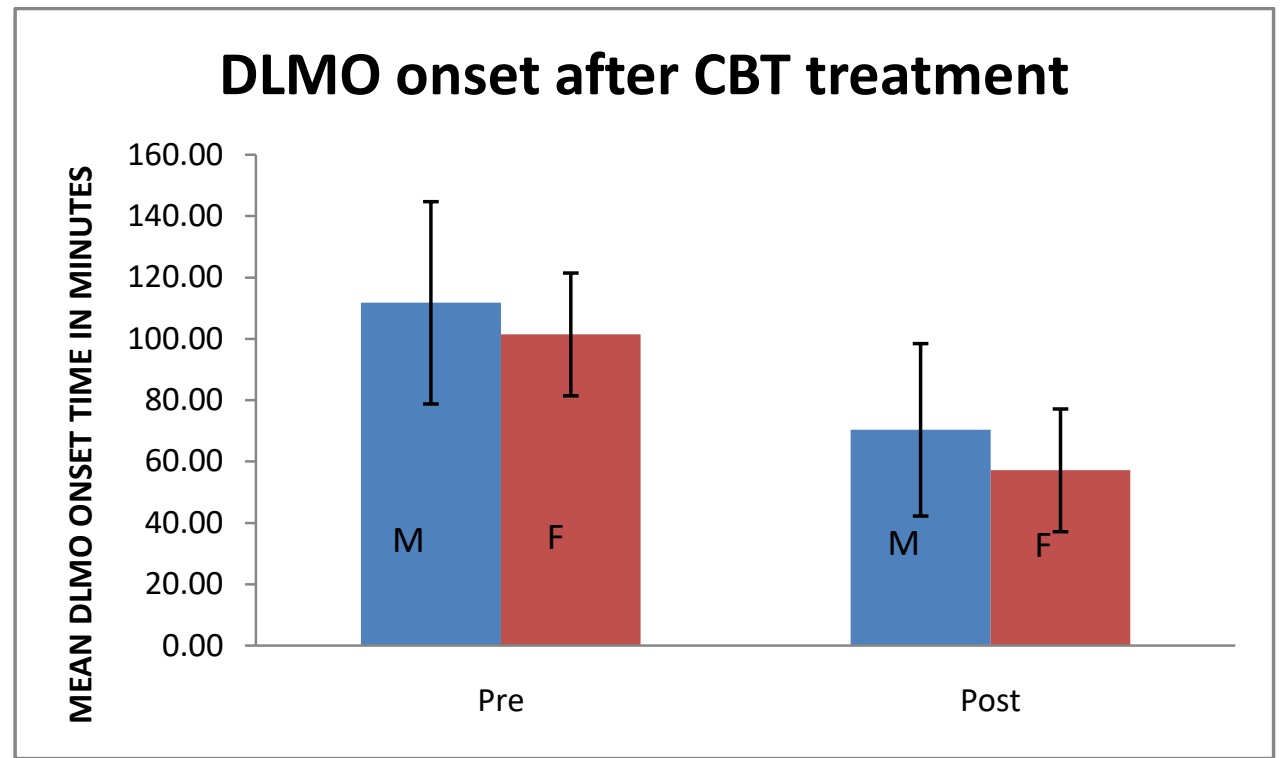

Figure 1: Mean DImo Onset Time in Minutes dor Males Vs Females Before and after Intervention 